\title{
Predictors of Outcome in Intracerebral Hemorrhage and Performance of Hemphill ICH Score and GVS Score
}

\author{
Mahmudul Islam', Mashfiqul Hasan², Sudipta Kumer Mukherjee ${ }^{3}$, Jobaida Naznin, \\ Rafiqul Islam 5 , Maliha Hakim ${ }^{6}$
}

${ }^{1}$ Assistant Professor, Department of Neurology, National Institute of Neurosciences \& Hospital, Dhaka, Bangladesh; ${ }^{2}$ Assistant Professor of Endocrinology, National Institute of Neurosciences and Hospital, Dhaka, Bangladesh; ${ }^{3}$ Assistant Professor, Department of Pediatric Neurosurgery, National Institute of Neurosciences and Hospital, Dhaka, Bangladesh; ${ }^{4}$ Assistant Professor, Department of Endocrinology, Shaheed Suhrawardy

Medical College, Dhaka, Bangladesh; ${ }^{5}$ Associate Professor, Department of Pharmacology, National Institute of Neurosciences \& Hospital, Dhaka, Bangladesh; ${ }^{6}$ Professor, Department of Neurology, National Institute of Neurosciences \& Hospital, Dhaka, Bangladesh

[Received on: 22 November 2020; Accepted on: 12 December 2020; Published: 1 January 2021]

\begin{abstract}
Background: The outcome of intracerebral hemorrhage (ICH) is gloomy. There are several scoring systems for predicting its outcome. Objective: The purpose of the present study was to observe the predictors of outcome in ICH patients and to assess the performance of 'Hemphill ICH score' and 'GVS score'. Methodology: This cohort study involved patients of ICH admitted within 72 hours of acute event in July to December 2017 in the Department of Neurology at National Institute of Neurosciences (NINS) \& Hospital, Dhaka, Bangladesh. Clinical and radiological data at admission and in-hospital events were obtained from medical records. Patients who were discharged from the hospital were interviewed at 30th day after event by face to face interview or over telephone. Follow up data was not found for 4 patients. Results: This study involved 115 patients of ICH [median age 60 years (interquartile range, IQR 50-70); 46\% (53/115) female]. In-hospital and 30-day mortality of the ICH patients was 22\% (25/115: 95\% CI 15-30\%) and 38\% (42/111; 95\% CI 29-48\%) respectively. There was almost perfect agreement between Hemphill ICH score and GVS score $(p<0.001$ and $\kappa=0.862)$. Kaplan-Meier survival curves of patients with different Hemphill ICH scores revealed significant difference among them; which was also true for patients with different GVS scores (by log-rank test, $\mathrm{p}<0.001$ for both). The area under the curve (AUC) for the Hemphill ICH score to predict mortality was $0.70(95 \% \mathrm{CI}, 0.60-0.80)$ and for the GVS score 0.74 (95\% CI, 0.64-0.83). In bivariate logistic regression, NIHSS score, GCS score, blood glucose at admission, nosocomial pneumonia, ICH volume, midline shift along with both Hemphill ICH and GVS score were observed to predict mortality at 30 days ( $<0.05$ for all). In two separate models adjusting for blood glucose and nosocomial pneumonia, every 1-point increase in the Hemphill ICH score and GVS score increases the mortality risk by 2.35 fold (adjusted OR: $2.35 ; 95 \%$ CI 1.33-4.16; $\mathrm{p}=0.003$ ) and 2.99 fold (adjusted OR: 2.99; 95\% CI 1.57-5.72; $\mathrm{p}=0.001$ ) respectively. Conclusions: Both Hemphill ICH and GVS score have comparable predicting ability of outcome in ICH. In addition to components of scoring systems, occurrence of nosocomial pneumonia and blood glucose seems important. [Journal of National Institute of Neurosciences Bangladesh, January 2021;7(1): 3-9]
\end{abstract}

\section{Keywords: Intracerebral hemorrhage; Hemphill ICH score; GVS score}

Correspondence: Dr. Mahmudul Islam, Assistant Professor, Department of Neurology, National Institute of Neurosciences and Hospital, Sher-E-Bangla Nagar, Agargaon, Dhaka-1207, Bangladesh; Email: drmahmudul@yahoo.com; Cell no: +8801822496267 Conflict of interest: There is no conflict of interest relevant to this paper to disclose.

Funding agency: This research project was not funded by any group or any institution.

Contribution to authors: MI \& MH were involved in protocol preparation, MH \& JN were involved in data collection and literature search, MI, MH \& MH were involved in manuscript writing. MH \& SKM were involved in preparation and revision of this manuscript.

How to cite this article: Islam M, Hasan M, Mukherjee SK, Naznin J, Islam R, Hakim M. Predictors of Outcome in Intracerebral Hemorrhage and Performance of Hemphill ICH Score and GVS Score. J Natl Inst Neurosci Bangladesh, 2021;7(1): 3-9

Copyright: (C2021. Islam et al. Published by Journal of National Institute of Neurosciences Bangladesh. This article is published under the Creative Commons CC BY-NC License (https://creativecommons.org/licenses/by-nc/4.0/). This license permits use, distribution and reproduction in any medium, provided the original work is properly cited, and is not used for commercial purposes.

\section{Introduction}

Stroke is recognised as an important cause of mortality and morbidity worldwide as it is the second most common cause of death and the third most common 
cause of disability ${ }^{1-2}$. Broadly there are two subcategories of stroke: ischemic and hemorrhagic. About $10.0 \%$ to $15.0 \%$ of stroke is hemorrhagic which is again subdivided into hemorrhage into brain parenchyma known as intracerebral haemorrhage (ICH) and hemorrhage into subarachnoid space known as subarachnoid haemorrhage $(\mathrm{SAH})^{3}$.

Among the different types of stroke, ICH is more common in low income countries and has a higher risk of morbidity and mortality than ischemic stroke and $\mathrm{SAH}^{3,4}$. It has a 30 -days mortality of around $40 \%$ and worldwide it remains a big concern for neuroscientists ${ }^{5}$. To predict the prognosis of ICH it is important to know which factors are related to outcome. There have been a number of prediction models that have been developed for $\mathrm{ICH}^{4,6-12}$. Among them, most widely used prediction model is the Hemphill ICH score developed by Hemphill et $\mathrm{al}^{4}$.

According to Hemphill ICH scoring system, independent predictors for 30-days mortality in ICH are greater ICH volume (score 0 if $<30 \mathrm{~cm}^{3}$, score 1 if $\geq 30 \mathrm{~cm}^{3}$ ) ,infratentorial location of ICH (score 0 if supratentorial, score 1 if infratentorial), low score on Glasgow Coma Scale (GCS) (score 0 if 13-15, score 1 if 5-12, score 2 if $3-4$ ), older age (score 0 if $<80$ years, score 1 if $\geq 80$ years), and intraventricular extension of the hemorrhage (score 0 if no, score 1 if yes) ${ }^{4}$. On the other hand, Mukherjee et al. proposed a simplified model for prediction of prognosis of ICH and named it as 'GVS score" ${ }^{6}$. The 'GVS' represents the three components of the scoring system where ' $G$ ' stands for GCS score (score 0 if $13-15$, score 1 if $5-12$, score 2 if 3-4), ' $V$ ' for volume of hemorrhage (score 0 if $<30 \mathrm{~cm}^{3}$, score 1 if $\geq 30$ $\mathrm{cm} 3$ ) and ' $\mathrm{S}$ ' for site of hemorrhage (score 0 if supratentorial, score 1 if intraventricular or cerebellar and score 2 if brain stem). Despite its simplicity, the GVS score was observed to have substantial agreement with Hemphill ICH score ${ }^{6}$. Following the initial study of the authors proposing GVS score, it has not been validated by further studies. In this background, the present study was carried out to observe the predictors of outcome in ICH patients and to assess the performance of Hemphill ICH as well as GVS score.

\section{Methodology}

This was a retrospective cohort study carried out through review of medical records of stroke patients admitted during July 2017 to December 2017 in a single unit of the Department of Neurology of National Institute of Neurosciences (NINS) \& Hospital, Dhaka, Bangladesh. The patients with acute ICH who presented within 72 hours of event and confirmed by computed tomography (CT) scan were enrolled. Clinical and radiological data recorded at admission were noted. ICH volume was measured on the initial CT scan of brain by using $\mathrm{ABC} / 2$ method. In this method $\mathrm{A}$ was the greatest diameter on the largest hemorrhage slice, $\mathrm{B}$ was the diameter perpendicular to $\mathrm{A}$ and $\mathrm{C}$ was the number of axial slices with hemorrhage multiplied by the slice thickness ${ }^{13}$. Patients were monitored for in-hospital events like surgical intervention, complications or death. Patients who were discharged from the hospital
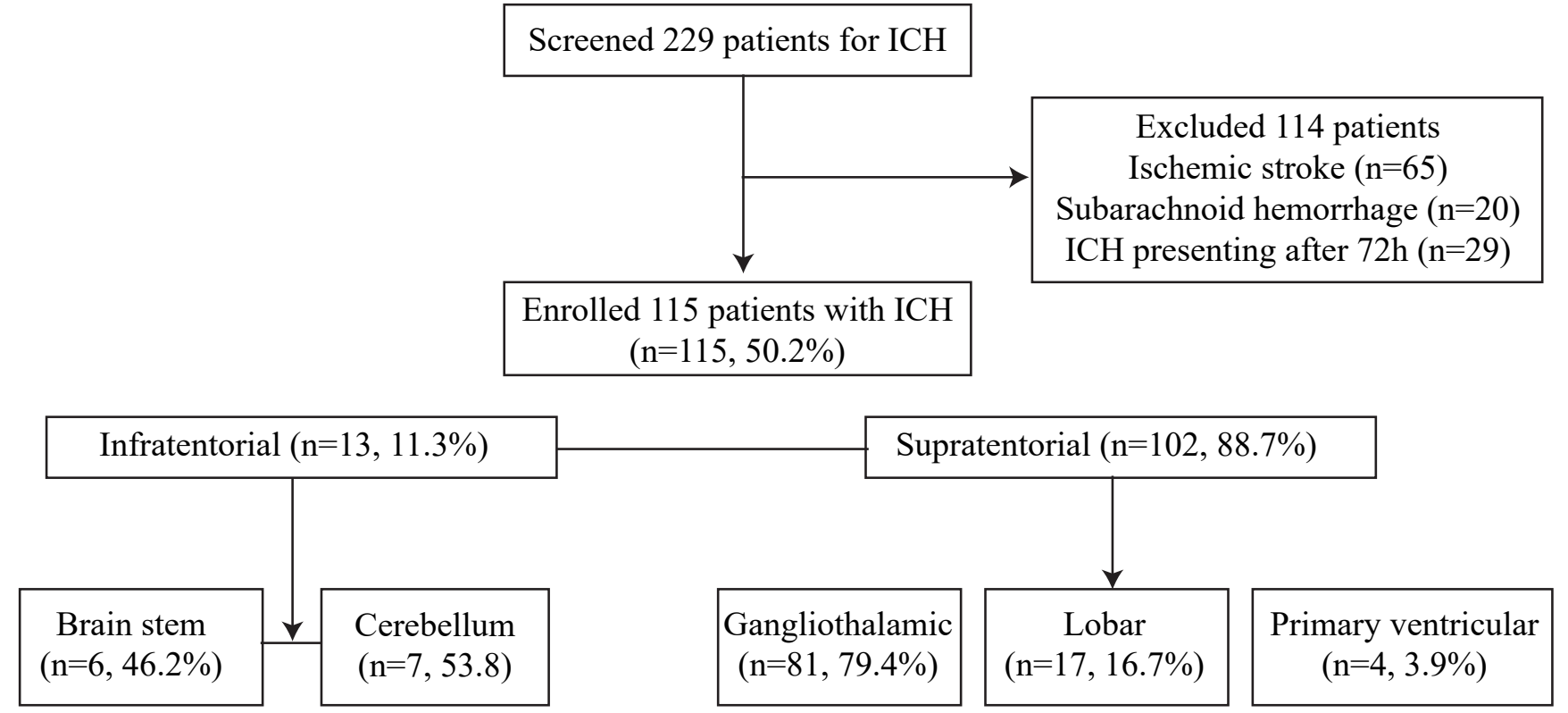

Figure I: The scheme of enrollment and radiological subtypes of ICH (ICH: Intracerebral hemorrhage) 
were followed up at $30^{\text {th }}$ day after the stroke event. Those who failed to attend NINS were followed up over telephone for modified Rankin Scale (mRS) score. The study was approved by the local ethical committee. Statistical analysis was performed by using Statistical Packages for Social Sciences (SPSS 22.0) (SPSS Inc, Chicago, IL, USA). Quantitative data were expressed as mean and standard deviation or median and interquartile range; and qualitative data were expressed as frequency and percentage. Normality of data was tested by Kolmogorov-Smirnov and Shapiro-Wilk test. $95 \%$ confidence limit was taken. Agreement of Hemphill ICH and GVS score was assessed by kappa test. Kaplan-Meier survival curve was constructed for participants of the study with different points in Hemphill ICH and GVS score separately and log-rank test was done to compare the difference in survival. Bivariate logistic regression was done to evaluate the predictors of 30-day mortality. Thereafter two separate multivariate logistic regressions adjusted for significant predictors in bivariate model was done to evaluate the Hemphill ICH score and GVS score as predictors. Receiver operating characteristic (ROC) curve was used to evaluate the performance of both the scores.

\section{Results}

A total number of 229 patients were screened during the study period and among them 115 of CT-confirmed ICH patients were included (Figure I).

The median age of the participants was 60 (IQR 50-70) years and 46 (53/115) were female. Their median Hemphill ICH score as well as GVS score was 2 (IQR 2-3 \& 1-3 for Hemphill ICH and GVS score respectively). Median hospital stay was 6 (IQR-5-7) days and in-hospital mortality was 22\% (25/115: $95 \%$ CI 15-30\%) (Table 1).
Table 1: Demographic and clinical parameters of the study participants $(\mathrm{n}=115)$

\begin{tabular}{lc}
\hline Characteristics & Frequency \\
\hline Age (years; median and IQR) & $60(50-70)$ \\
Sex $\quad$ Male $\quad$ Female & $62(53.9)$ \\
& $53(46.1)$ \\
History of hypertension & $89(77.4)$ \\
History of diabetes mellitus & $20(17.4)$ \\
Previous stroke & $12(10.4)$ \\
Family history of stroke & $25(21.7)$ \\
History of cardiac disease & $6(5.2)$ \\
History of smoking & $32(27.8)$ \\
Duration of symptoms (hours; median and IQR) & $24(10-48)$ \\
NIHSS score on admission (median and IQR) & $22(14-27)$ \\
GCS score on admission (median and IQR) & $8(7-12)$ \\
Systolic BP (mm Hg; median and IQR) & $160(145-180)$ \\
Diastolic BP (mm Hg; median and IQR) & $100(80-100)$ \\
Blood glucose at admission (mmol/L; median & $8.3(6.9-10.2)$ \\
and IQR) & \\
Pre-event mRS score & $0(0-0)$ \\
Hemphill ICH score (median and IQR) & $2(2-3)$ \\
GVS score (median and IQR) & $2(1-3)$ \\
Nosocomial pneumonia & $12(10.4)$ \\
Surgical measures & $4(3.5)$ \\
Hospital stay (days; median and IQR) & $6(5-7)$ \\
In-hospital Mortality & $25(21.7)$ \\
&
\end{tabular}

Within parentheses are percentages over column total if not mentioned otherwise; IQR: Interquartile range; NIHSS: National Institute of Health Stroke Scale; GCS: Glasgow Coma Scale; mRS: modified Rankin scale

Among the study participants, 4 out of 115 (3.5\%) had no follow up data at 30 days. In the remaining 111 participants, the 30-day case fatality was 38\% (42/111); $95 \%$ CI $29-48 \%$. The 30 -day case fatality for patients with a Hemphill ICH score of $0,1,2,3$ and 4 was $0.0 \%$,

Table 2: Agreement of Hemphill ICH score and GVS score

\begin{tabular}{|c|c|c|c|c|c|c|c|}
\hline & & \multicolumn{5}{|c|}{ GVS score } & \multirow[t]{2}{*}{ Total } \\
\hline & & 0 & 1 & 2 & 3 & 4 & \\
\hline \multicolumn{8}{|c|}{ Hemphill ICH score } \\
\hline & 0 & $3(100.0)$ & - & - & - & - & 3 \\
\hline & 1 & - & $25(96.2)$ & - & - & - & 25 \\
\hline & 2 & - & $1(3.8)$ & $40(90.9)$ & $3(7.7)$ & - & 44 \\
\hline & 3 & - & - & $4(9.1)$ & $33(84.6)$ & - & 37 \\
\hline & 4 & - & - & - & $3(7.7)$ & $3(100.0)$ & 6 \\
\hline Total & & 3 & 26 & 44 & 39 & 3 & \\
\hline
\end{tabular}

$\mathrm{p}<0.001, \kappa=0.862$; within parentheses are percentages over column total 
$12.0 \%, 37.0 \%, 54.0 \%$ and $67.0 \%$ respectively. On the other hand, the 30-day case fatality for patients with an GVS score of $0,1,2,3$ and 4 was $0.0 \%, 12.0 \%, 33.0 \%$, $58.0 \%$ and $100.0 \%$ respectively (Figure II). There was almost perfect agreement between Hemphill ICH score and GVS score $(\mathrm{p}<0.001$ and $\kappa=0.862)$ (Table 2$)$.

Kaplan-Meier survival curves of patients with different Hemphill ICH scores revealed significant difference among them; which was also true for patients with different GVS scores (by log-rank test, $\mathrm{p}<0.001$ for both) (Figure III).

In bivariate logistic regression, National Institute of Health Stroke Scale (NIHSS) score, GCS score and blood glucose at admission, nosocomial pneumonia, ICH volume, midline shift along with both Hemphill

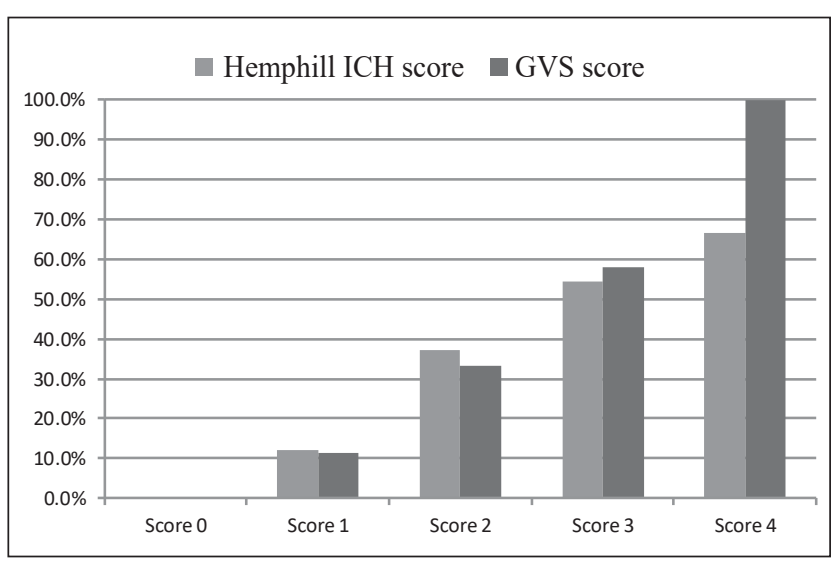

Figure II: Case fatality of the intracerebral hemorrhage patients $(n=111)$ at 30 days stratified by Hemphill ICH score and GVS score (for Hemphill ICH score 0, 1, 2, 3, 4: n= 2, 25, 43, 35, 6 and for GVS score $0,1,2,3,4: \mathrm{n}=2,26,42,38,3$ respectively; no patients had Hemphill ICH score 5-6 or GVS score 5)
ICH and GVS score were observed to predict mortality at 30 days in the participants $(\mathrm{p}<0.05)$ (Table 3 ).

Table 3: Predictors of prognosis (30-day mortality) including ICH and GVS score by bivariate logistic regression

\begin{tabular}{lcc}
\hline Variables & OR (95\% CI) & P value \\
\hline Age, per year increase & $1.02(0.98-1.05)$ & 0.370 \\
$\begin{array}{l}\text { Female sex } \\
\text { Systolic blood pressure, }\end{array}$ & $0.77(0.36-1.67)$ & 0.511 \\
per mm of Hg increase & $1.00(0.99-1.02)$ & 0.747 \\
$\begin{array}{l}\text { Diastolic blood pressure, } \\
\text { per mm of Hg increase }\end{array}$ & $1.01(0.99-1.03)$ & 0.485 \\
Admission NIHSS score, & & \\
per unit increase & $1.26(1.15-1.38)$ & $<\mathbf{0 . 0 0 1}$ \\
Admission GCS score, & & \\
per unit increase & $0.65(0.53-0.78)$ & $<\mathbf{0 . 0 0 1}$ \\
Random blood glucose, & & \\
per mmol increase & $1.27(1.10-1.47)$ & $\mathbf{0 . 0 0 1}$ \\
White cell count, & & \\
per unit increase & $1.02(0.91-1.14)$ & $\mathbf{0 . 7 6 7}$ \\
$\begin{array}{l}\text { Prior history of hypertension } \\
\text { Prior history of diabetes }\end{array}$ & $1.18(0.45-3.08)$ & $\mathbf{0 . 7 3 5}$ \\
Time from symptom onset to hospital & $1.24(0.45-3.39)$ & $\mathbf{0 . 6 7 4}$ \\
presentation, per hour increase & $1.01(0.99-1.02)$ & 0.603 \\
Hyponatremia & $1.44(0.54-3.83)$ & 0.467 \\
$\begin{array}{l}\text { Renal impairment } \\
\text { Nosocomial pneumonia }\end{array}$ & $2.03(0.86-4.77)$ & 0.104 \\
ICH volume, per cm ${ }^{3}$ increase & $10.47(2.17-50.60)$ & 0.029 \\
Presence of IVH & $1.02(1.01-1.02)$ & $<\mathbf{0 . 0 0 1}$ \\
Midline shift, per mm increase & $1.29(0.58-2.87)$ & 0.540 \\
Infratentorial hemorrhage & $1.25(1.12-1.39)$ & $<\mathbf{0 . 0 0 1}$ \\
Hemphill ICH score & $0.45(0.12-1.75)$ & 0.252 \\
GVS score & $2.52(1.51-4.20)$ & $<\mathbf{0 . 0 0 1}$ \\
\hline & $3.42(1.91-6.12)$ & $<\mathbf{0 . 0 0 1}$ \\
\hline
\end{tabular}

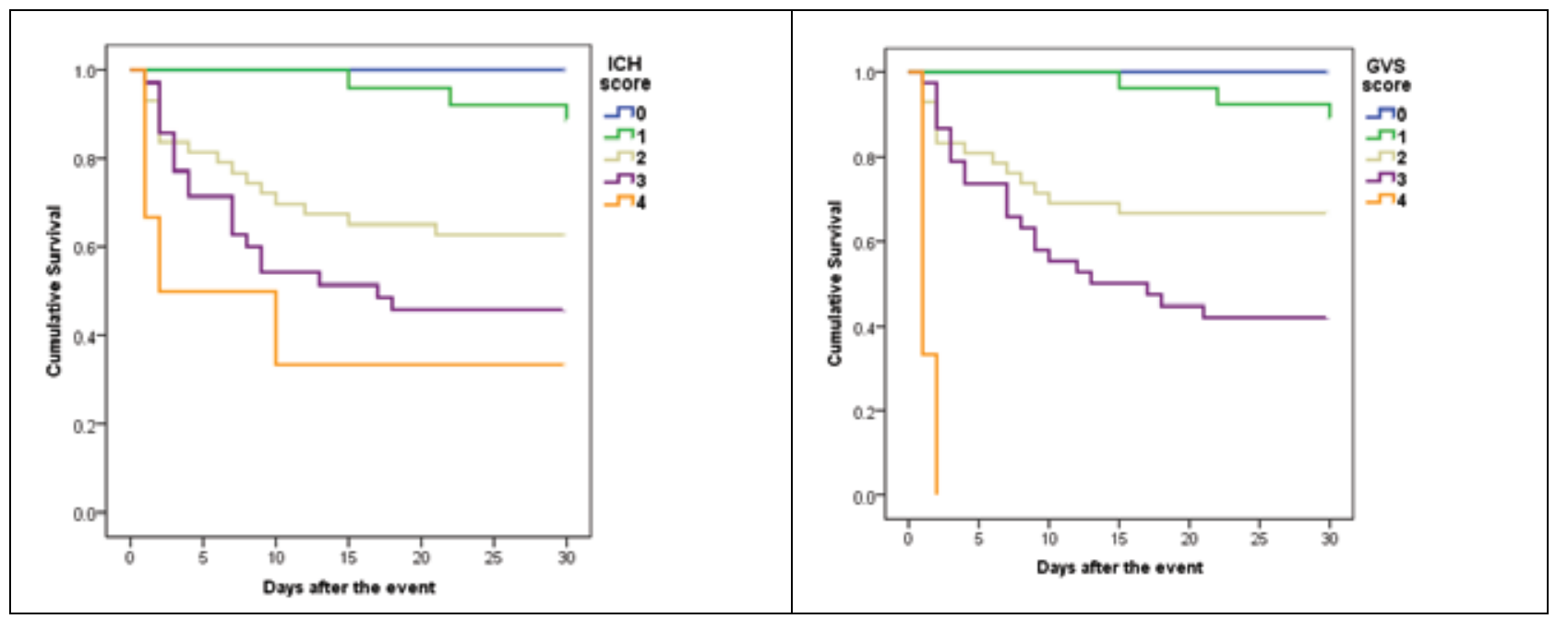

Figure III: Kaplan-Meier survival curve of patients with different Hemphill ICH score and GVS score (left and right panel respectively) revealed significant difference among them (by log-rank test, $\mathrm{p}<0.001$ test for both) 
In a model adjusting for blood glucose and aspiration pneumonia, it was observed that there is 2.35 -fold increase in mortality risk for every 1-point increase in the Hemphill ICH score (adjusted OR: 2.35; 95\% CI $1.33-4.16 ; \mathrm{p}=0.003)$. In another model with same adjustment, we observed 2.99 -fold increase in mortality for every 1-poit increase in the GVS score (adjusted OR: 2.99; 95\% CI 1.57-5.72; $\mathrm{p}=0.001$ ) (Table 4).

Table 4: Hemphill ICH score and GVS score in prediction of 30-day mortality when adjusted for blood glucose and pneumonia in two separate model of multivariate logistic regression

\begin{tabular}{lccc}
\hline Scores & ${ }^{*} \mathbf{R 2}$ & aOR $(\mathbf{9 5 \%} \mathbf{C I})$ & P value \\
\hline Hemphill ICH score & 0.290 & $2.35(1.33-4.16)$ & 0.003 \\
GVS score & 0.313 & $2.99(1.57-5.72)$ & 0.001 \\
\hline
\end{tabular}

${ }^{*}$ Cox and Snell

The area under the curve (AUC) for the Hemphill ICH score to predict mortality was $0.70(95 \% \mathrm{CI}$, $0.60-0.80)$, and for the GVS score was 0.74 (95\% CI, 0.64-0.83) (Figure IV).

\section{Discussion}

The current study evaluated the in-hospital and 30-day mortality of ICH patients admitted within 72-hours of event in a referral neuroscience institute of Dhaka and observed $22.0 \%$ and $38.0 \%$ mortality respectively. Along with increasing Hemphill ICH and GVS score, lower GCS score, higher blood glucose at admission, pneumonia, higher ICH volume and midline shift in CT scan were observed to be associated with higher mortality at 30 days. There was almost perfect agreement between Hemphill ICH and GVS score. Despite its simplicity, the GVS score AUC was not below Hemphill ICH score.

In spite of advancements in medical service, case fatality rate of ICH patients is not decreasing worldwide 5 . Outcome of ICH patients in our study was comparable to those found by different studies in home and abroad ${ }^{14-16}$. However, our study was conducted in the in-patient department of a neuroscience institute situated in the capital of the country and so it may be generalizable to only those who present to this hospital for care. GCS score was observed to be a good predictor of mortality in ICH patients by both present and previous studies ${ }^{9,14,17,18}$, though it was primarily developed for evaluating patients of head injury. As a result, GCS score is invariably incorporated in popular ICH outcome predicting scales. Despite its limitations especially in aphasic patient, GCS remains a simple and reliable tool to assess outcome of ICH patients. NIHSS score originally used for assessing the severity of ischemic stroke, was also observed to be a good predictor of outcome in current as well as in other studies ${ }^{19,20}$. However, it takes a few minutes to assess NIHSS score and it may not be as simple as applying GCS at bedside.

Initial CT scan of brain has an important role in predicting outcome of patients with ICH. Volume of hematoma was unequivocally seen to be associated with mortality $9,14,17,18,21$; which is also true for our study. Although it was observed that $\mathrm{ABC} / 2$ formula tends to overestimate intracerebral hematoma volume in comparison to computer-assisted volumetric analysis

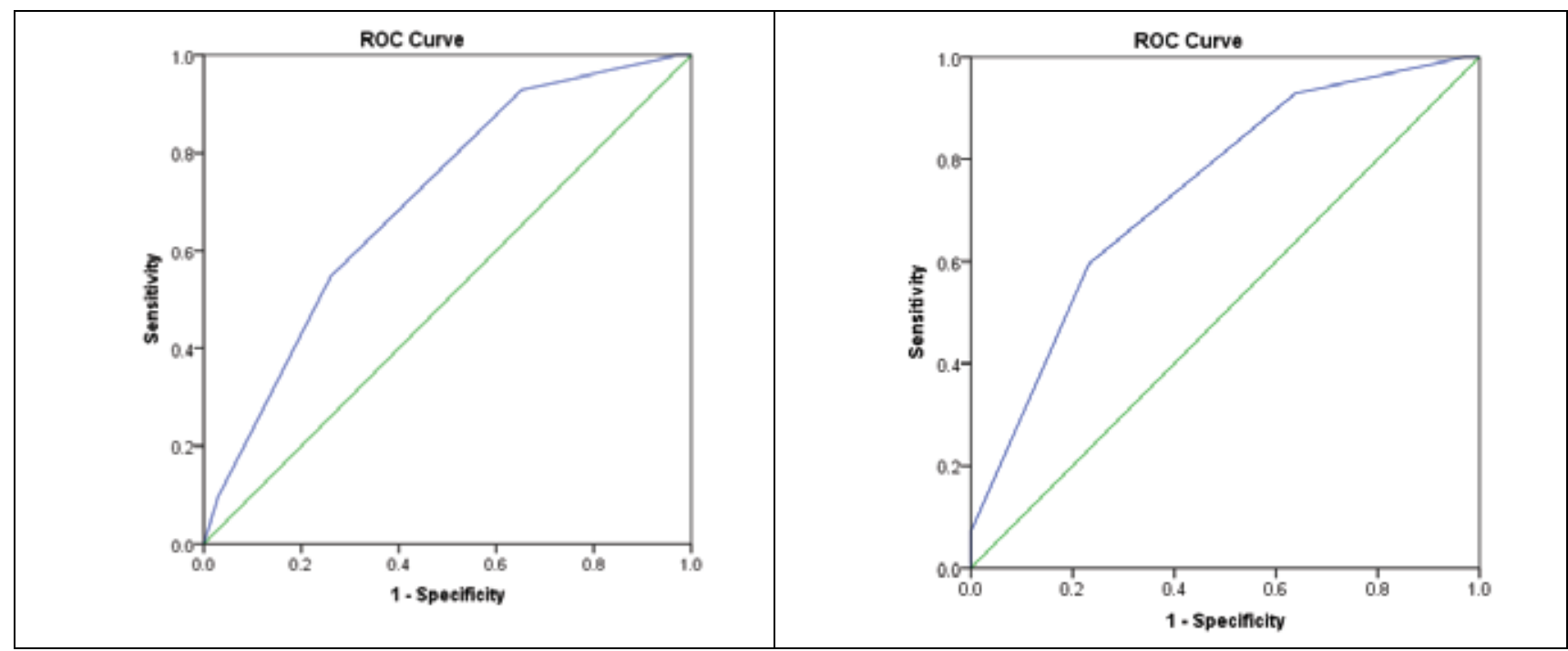

Figure IV: Receiver operating characteristic (ROC) curve for prediction of mortality in patients with intracerebral hemorrhage: left panel Hemphill ICH score, right panel GVS score 
$(\mathrm{CAVA})^{22}$, it is still useful as an rapid and reliable method $^{23}$. Intraventricular hemorrhage (IVH) adds a point in both Hemphill ICH and GVS score and has been considered to be a poor prognostic factor in various studies ${ }^{9,15,16}$. But the present study as well as some other studies could not appreciate its relationship to ICH outcome ${ }^{18,24}$. Faigle et $\mathrm{al}^{25}$ observed a differential impact of IVH over ICH outcome in different races. Indeed, few authors opined that ICH with ventricular extension and no hydrocephalus may not increase mortality or severe disability ${ }^{24}$. So the direct relationship of IVH to outcome in ICH is not often observed. Mass effect of the hematoma induces neuronal injury; and midline shift in CT scan of brain is a simple way to quantify it. The present study also observed higher mortality in patients with higher midline shift. While it is acknowledged that midline shift is associated with poor outcome in patients with $\mathrm{ICH}^{26}$, it has not been included in either ICH or GVS score. One explanation may be the relationship of hematoma volume measurement with midline shift as replacing hematoma volume with midline shift in ICH score resulted almost similar predictive capacity ${ }^{27}$. Site of hemorrhage in ICH is also included in both Hemphill ICH and GVS score and infratentorial location had been observed to be strongly related to mortality ${ }^{9,18,28}$. However, hemorrhage into the brain stem or cerebellum does not carry equal risk. Brain stem hemorrhage is particularly observed to be related to mortality ${ }^{29}$ and hence it is given more weight in GVS score. The current study could not relate it to outcome probably due to small proportion of patients in infratentorial hemorrhage group $(13 / 115 ; 11 \%)$.

Nosocomial pneumonia is a frequent complication among ICH patients. We observed around one in ten patients of ICH developed pneumonia, rate of which is comparable to previous studies ${ }^{30,31}$. Likewise, mortality in ICH patients with pneumonia was also higher in our as well as in other studies ${ }^{32}$. Occurrence of pneumonia may add more predicting power for negative outcome in ICH patients and may be considered while modifying the current scoring scales. Blood glucose is also not included in any of the predicting scores and studies did not confirm a strong and consistent association $^{33-37}$. The present study observed an association of higher blood glucose at admission to mortality which is supported by a meta-analysis published in $2014^{38}$. In multivariate regression analysis we adjusted for pneumonia and blood glucose in two separate models for predicting ability of ICH and GVS score. Both the scores had good predicting ability with increase in one-point score result in more than two-fold increase chance of mortality. There was almost perfect agreement of ICH and GVS score with comparable AUC and mortality after 30 days of event. It seems that both the scores are good at predicting outcome of ICH patient. Due to its simplicity GVS score may be strongly considered in our setting.

\section{Conclusion}

In conclusion, both ICH and GVS score have comparable predicting ability of outcome in ICH. In addition to components of scoring systems, occurrence of nosocomial pneumonia and blood glucose seems important in our setting.

\section{References}

1. Thrift AG, Thayabaranathan T, Howard G, Howard VJ, Rothwell PM, Feigin VL, Norrving B, Donnan GA, Cadilhac DA. Global stroke statistics. International Journal of Stroke. 2017;12(1):13-32.

2. Feigin VL, Forouzanfar MH, Krishnamurthi R, Mensah GA, Connor M, Bennett DA, Moran AE, Sacco RL, Anderson L, Truelsen T, O'Donnell M. Global and regional burden of stroke during 1990-2010: findings from the Global Burden of Disease Study 2010. The Lancet. 2014;383(9913):245-55

3. Langhorne P. Stroke Medicine. In: Ralston SH, Penman ID, Strachan MWJ, Hobson RP, eds. Davidson's princeples and practice of Medicine. 23rd Edition, Elsevier, 2018.

4. Hemphill JC, Bonovich DC, Besmertis L, Manley GT, Johnston SC. The ICH score. Stroke. 2001;32(4):891-7.

5. Van Asch CJ, Luitse MJ, Rinkel GJ, van der Tweel I, Algra A, Klijn CJ. Incidence, case fatality, and functional outcome of intracerebral haemorrhage over time, according to age, sex, and ethnic origin: a systematic review and meta-analysis. The Lancet Neurology. 2010;9(2):167-76.

6. Mukherjee SK. The GVS: Glasgow coma score, volume \& site of hemorrhage as a simple scoring system for intracerebral hemorrhage. SOJ Neurol. 2016;3(1):1-5.

7. Weimar C, Benemann J, Diener HC. Development and validation of the Essen intracerebral haemorrhage score. Journal of Neurology, Neurosurgery \& Psychiatry. 2006;77(5):601-5.

8. Parry-Jones AR, Abid KA, Di Napoli M, Smith CJ, Vail A, Patel HC, King AT, Tyrrell PJ. Accuracy and clinical usefulness of intracerebral hemorrhage grading scores: a direct comparison in a UK population. Stroke. 2013;44(7):1840-5.

9. Zis P, Leivadeas P, Michas D, Kravaritis D, Angelidakis P, Tavernarakis A. Predicting 30-day case fatality of primary inoperable intracerebral hemorrhage based on findings at the emergency department. Journal of Stroke and Cerebrovascular Diseases. 2014;23(7):1928-33.

10. Rost NS, Smith EE, Chang Y, Snider RW, Chanderraj R, Schwab K, FitzMaurice E, Wendell L, Goldstein JN, Greenberg SM, Rosand J. Prediction of functional outcome in patients with primary intracerebral hemorrhage: the FUNC score. Stroke. 2008;39(8):2304-9.

11. Ariesen MJ, Algra A, Van der Worp HB, Rinkel GJ. Applicability and relevance of models that predict short term outcome after intracerebral haemorrhage. Journal of Neurology, Neurosurgery \& Psychiatry. 2005;76(6):839-44.

12. Cheung RT, Zou LY. Use of the original, modified, or new 
intracerebral hemorrhage score to predict mortality and morbidity after intracerebral hemorrhage. Stroke. 2003;34(7):1717-22.

13. Kothari RU, Brott T, Broderick JP, Barsan WG, Sauerbeck LR, Zuccarello M, Khoury J. The ABCs of measuring intracerebral hemorrhage volumes. Stroke. 1996;27(8):1304-5.

14. Mondal RN, Barman S, Islam MJ, Jahan SM, Alam AM, Hussain MM, Sarker MK, Rahman MM, Sarkar H, Rani M, Hossain J. Short-term predictors of mortality among patients with hemorrhagic stroke. World Heart Journal. 2014;6(4):273.

15. Bhatia R, Singh H, Singh S, Padma MV, Prasad K, Tripathi M, Kumar G, Singh MB. A prospective study of in-hospital mortality and discharge outcome in spontaneous intracerebral hemorrhage. Neurology India. 2013;61(3):244.

16. Narayan SK, Sivaprasad P, Sushma S, Sahoo RK, Dutta TK. Etiology and outcome determinants of intracerebral hemorrhage in a south Indian population, A hospital-based study. Annals of Indian Academy of Neurology. 2012;15(4):263.

17. Wang CW, Liu YJ, Lee YH, Hueng DY, Fan HC, Yang FC, Hsueh CJ, Kao HW, Juan CJ, Hsu HH. Hematoma shape, hematoma size, Glasgow coma scale score and ICH score: which predicts the 30-day mortality better for intracerebral hematoma?. PLoS One. 2014;9(7):e102326

18. Safatli DA, Günther A, Schlattmann P, Schwarz F, Kalff R, Ewald C. Predictors of 30-day mortality in patients with spontaneous primary intracerebral hemorrhage. Surgical neurology international. 2016;7(Suppl 18):S510.

19. Cheung CM, Tsoi TH, Hon SF, Au-Yeung M, Shiu KL, Lee CN, Huang CY. Using the National Institutes of Health Stroke Scale (NIHSS) to predict the mortality and outcome of patients with intracerebral haemorrhage. Hong Kong Med J. 2008;14(5):367-70.

20. Mahdy ME, Ghonimi NA, Elserafy TS, Mahmoud W. The NIHSS score can predict the outcome of patients with primary intracerebral hemorrhage. The Egyptian Journal of Neurology, Psychiatry and Neurosurgery. 2019;55(1):21.

21. Abdallah A, Chang JL, O'Carroll CB, Okello S, Olum S, Acan M, Aden AA, Chow FC, Siedner MJ. Validation of the Intracerebral Hemorrhage Score in Uganda: A prospective cohort study. Stroke. 2018;49(12):3063-6.

22. Wang CW, Juan CJ, Liu YJ, Hsu HH, Liu HS, Chen CY, Hsueh CJ, Lo CP, Kao HW, Huang GS. Volume-dependent overestimation of spontaneous intracerebral hematoma volume by the ABC/2 formula. Acta Radiologica. 2009;50(3):306-11.

23. Tsair TJ, Aziz A. Reproducibility and accuracy of ABC/2 and $3 \mathrm{D}$ techniques in ICH volume measurement. Radiography. 2010;16(3):209-16.

24. Mahta A, Katz PM, Kamel H, Azizi SA. Intracerebral hemorrhage with intraventricular extension and no hydrocephalus may not increase mortality or severe disability. Journal of Clinical Neuroscience. 2016;30:56-9

25. Faigle R, Marsh EB, Llinas RH, Urrutia VC, Gottesman RF. Race-Specific Predictors of Mortality in Intracerebral Hemorrhage: Differential Impacts of Intraventricular Hemorrhage and Age Among Blacks and Whites. Journal of the American Heart Association. 2016;5(8):e003540.

26. Krishnakumar P, Bhadran B, Harrison G, Aravind KR. Radiological predictors of mortality in patients with primary spontaneous intracerebral haemorrhage. Journal of Evolution of Medical and Dental Sciences. 2018;7(18):2192-7.

27. Yang WS, Li Q, Li R, Liu QJ, Wang XC, Zhao LB, Xie P. Defining the optimal midline shift threshold to predict poor outcome in patients with supratentorial spontaneous intracerebral hemorrhage. Neurocritical care. 2018;28(3):314-21.

28. Delcourt C, Sato S, Zhang S, Sandset EC, Zheng D, Chen X, Hackett ML, Arima H, Hata J, Heeley E, Salman RA. Intracerebral hemorrhage location and outcome among INTERACT2 participants. Neurology. 2017;88(15):1408-14.

29. Chen R, Wang X, Anderson CS, Robinson T, Lavados PM, Lindley RI, Chalmers J, Delcourt C, INTERACT Investigators. Infratentorial Intracerebral Hemorrhage: Relation of Location to Outcome. Stroke. 2019;50(5):1257-9.

30. Divani AA, Hevesi M, Pulivarthi S, Luo X, Souslian F, Suarez JI, Bershad EM. Predictors of nosocomial pneumonia in intracerebral hemorrhage patients: a multi-center observational study. Neurocritical care. 2015;22(2):234-42.

31. Maramattom BV, Weigand S, Reinalda M, Wijdicks EF, Manno EM. Pulmonary complications after intracerebral hemorrhage. Neurocritical care. 2006;5(2):115-9.

32. savi M, Doraiswamy V, Katapally R, Korya D, Adelman M, Miller R. Predictors and outcomes of pneumonia in patients with spontaneous intracerebral hemorrhage. Journal of intensive care medicine. 2013;28(2):118-23.

33. Dandapat S, Siddiqui FM, Fonarow GC, Bhatt DL, Xu H, Matsouaka R, Heidenreich PA, Xian Y, Schwamm LH, Smith EE. A paradoxical relationship between hemoglobin A1C and in-hospital mortality in intracerebral hemorrhage patients. Heliyon. 2019;5(5):e01659.

34. Chen R, Ovbiagele B, Feng W. Diabetes and stroke: epidemiology, pathophysiology, pharmaceuticals and outcomes. The American journal of the medical sciences. 2016;351(4):380-6 35. Wu TY, Putaala J, Sharma G, Strbian D, Tatlisumak T, Davis SM, Meretoja A. Persistent hyperglycemia is associated with increased mortality after intracerebral hemorrhage. Journal of the American Heart Association. 2017;6(8):e005760.

36. Al-Weshahy A, El-Sherif R, Selim KA, Heikal A. Short term outcome of patients with hyperglycemia and acute stroke. The Egyptian Journal of Critical Care Medicine. 2017;5(3):93-8.

37. Mapoure YN, Ba H, Ayeah CM, Kenmegne C, Luma HN, Njamnshi AK. Acute stroke patients with newly diagnosed diabetes mellitus have poorer outcomes than those with previously diagnosed diabetes mellitus. Journal of Stroke and Cerebrovascular Diseases. 2018;27(9):2327-35.

38. Guo X, Li H, Zhang Z, Li S, Zhang L, Zhang J, Han G. Hyperglycemia and mortality risk in patients with primary intracerebral hemorrhage: a meta-analysis. Molecular neurobiology. 2016;53(4):2269-75. 